\title{
A simple stochastic cellular automaton for synchronized traffic flow
}

\author{
Thorsten Chmura ${ }^{\mathrm{a}}$, Benedikt Herz ${ }^{\mathrm{b}}$, Florian Knorr ${ }^{\mathrm{c}, *}$, Thomas Pitz ${ }^{\mathrm{d}}$, \\ Michael Schreckenberg ${ }^{c}$

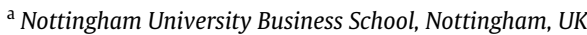 \\ ${ }^{\mathrm{b}}$ Universitat Pompeu Fabra, Barcelona, Spain \\ ' Physik von Transport und Verkehr, Universität Duisburg-Essen, 47048 Duisburg, Germany \\ ${ }^{\mathrm{d}}$ Rhine-Waal University of Applied Sciences, 47533 Kleve, Germany
}

\section{H I G H L I G H T S}

- This model is nearly as simple as the original NaSch.

- However, deceleration rates are restricted to realistic values.

- Therefore, it is free of collisions.

- The models show different velocity-synchronized phases.

\section{A R T I C L E I N F O}

\section{Article history:}

Received 2 September 2013

Received in revised form 24 February 2014

Available online 17 March 2014

\section{Keywords:}

Traffic flow

Cellular automaton

Synchronized flow

\begin{abstract}
A B S T R A C T
We present a very simple stochastic traffic cellular automaton (CA) model to reproduce synchronized traffic. This model aims to be nearly as simple as the wellknown Nagel-Schreckenberg model, but to overcome to shortcomings of the latter: The Nagel-Schreckenberg model (NaSch) and its variants achieve collision-free driving by explicitly allowing for unlimited braking capabilities. However, it is rather natural to view the collision-free traffic flow as a consequence of moderate driving instead of infinite braking capabilities. Therefore, our new CA model limits the vehicles' acceleration and deceleration rates to realistic values. Moreover, our model is able to reproduce several important features of synchronized traffic flow.

Earlier models achieve identical goals only by using relatively complicated rules of motion. Our model, however, introduces slight modifications of the easily comprehensible NaSch. These modifications lead to very stable states where all vehicles tend to synchronize their speed. Even though, or perhaps precisely because, the states of synchronized flow are stable in our model, it allows to study several features of this traffic phase in great detail.
\end{abstract}

(c) 2014 Elsevier B.V. All rights reserved.

\section{Introduction}

In 1992, Nagel and Schreckenberg [1] proposed one of the first stochastic discrete cellular automaton models of traffic on a one-lane road. Various modifications and extensions such as for the analysis of city traffic [2], the influence of traffic

\footnotetext{
* Corresponding author. Tel.: +49 203379 3901; fax: +49 2033796564.

E-mail addresses: thorsten.chmura@nottingham.ac.uk (T. Chmura), benedikt.herz@upf.edu (B. Herz), florian.knorr@uni-due.de (F. Knorr), thomas.pitz@hochschule-rhein-waal.de (T. Pitz), michael.schreckenberg@uni-due.de (M. Schreckenberg).
} 
lights [3], or the application to complex road networks [4,5] have been proposed since then (for reviews see, e.g., Refs. [6,7]). The primary criterion to assess these models is their ability to reproduce empirically observed features of traffic flow $[8,9]$, which follows from the vehicles' rules of motion.

In the Nagel-Schreckenberg model ( $\mathrm{NaSch}$ ), the road is modeled as a one-dimensional array of sites. Each site is occupied by at most one vehicle. The position of the $i$ th vehicle at time $t$ is denoted by $x_{t}^{i}$ and the position of its immediate predecessor by $x_{t}^{i+1}$. A vehicle's dynamics (i.e., its acceleration and deceleration) depends on its predecessor on the road. This behavior is implemented by an update scheme in which the following simple rules are applied to each vehicle in parallel:

1. A car $i$ with speed $v_{t}^{i}$ at time $t$ accelerates if the distance to its predecessor $\delta_{t}^{i}=x_{t}^{i+1}-x_{t}^{i}$ is sufficiently large. That means if $\delta_{t}^{i}$ is larger than $v_{t}^{i}+1$ then the speed is advanced by one until the car reaches its maximum speed $v_{\max }$ : $v_{t+1}^{i}=\min \left(v_{t}^{i}+1, v_{\max }\right)$.

2. If the distance $\delta_{t}^{i}$ is too small (i.e., $\left.\delta_{t}^{i} \leq v_{t+1}^{i}\right)$, the car reduces its speed to avoid a collision: $v_{t+1}^{i}=\min \left(\delta_{t}^{i}-1, v_{t+1}^{i}\right)$.

3. The car's speed is decreased by one at random: $v_{t+1}=\max \left(0, v_{t+1}^{i}-1\right)$ with probability $1-p_{\text {acc. }}$.

4. Finally, the vehicle moves from its current position $x_{t}^{i}$ to its new position $x_{t+1}^{i}=x_{t}^{i}+v_{t+1}^{i}$.

The success of the Nagel-Schreckenberg is based both on its simplicity and its ability to reproduce the spontaneous transition from free to congested flow revealing the stochastic nature of a traffic breakdown [10]. The latter is a result of the random deceleration of step 3 which leads to metastable states of free traffic flow. In this metastable regime, wide moving jams (i.e., waves of stopped vehicles) can spontaneously emerge from free flow (F). (Similar observations have been made for many modified versions of the NaSch [11-13].) Such a transition, however, conflicts with empirical observations: according to Kerner's three-phase theory ${ }^{1}$ of traffic $[14,15]$ congested traffic divides into two phases, synchronized traffic (S) and wide moving jams (W); a transition from free to congested traffic always involves a transition to synchronized traffic $(\mathrm{F} \rightarrow \mathrm{S})$. Therefore, the behavior observed in the NaSch and its successors $(\mathrm{F} \rightarrow \mathrm{J})$ does not correctly describe the physical characteristics of traffic flow.

The physical properties of the three traffic phases and the relation between them is essential for a correct understanding of traffic. In particular, the understanding of the synchronized phase $(S)$ is the key to any progress both in theoretical and application-oriented fields of research [16]. This insight has lead to the development of models that are based on Kerner's theory (e.g., Refs. [17-19]). These models successfully reproduce synchronized traffic and the corresponding transitions $(\mathrm{F} \leftrightarrow \mathrm{S}, \mathrm{S} \leftrightarrow \mathrm{J})$. At the same time, they are quite complex and involve an extensive set of rules.

In this paper, we will present a simple stochastic cellular automaton model that is able to reproduce synchronized traffic. Moreover, we achieve limited deceleration rates in our model by randomizing the acceleration step instead of the deceleration step (cf. step 3 of the NaSch). As a consequence, there is no metastable regime in our model. Even though, or perhaps precisely because, the states of synchronized flow are stable in our model, it allows to study several features of this traffic phase in great detail.

\section{Modified version of the NaSch}

First, we will present our modified model (in the following: mNaSch). Let $L \in \mathbb{N}$ be the number of sites representing the one-lane road. The discrete variable $t$ denotes the time.

At time $t$, the car labeled $i$ moves with speed $v_{t}^{i}$ which is bounded from above by $\mu\left(v_{t-1}^{i+1}, \delta_{t-1}^{i}\right)$. This upper boundary, whose value depends both on the speed of the leading vehicle and the distance gap, ensures that there are no collisions of two cars as we will show below. In our modified model, a car changes its speed according to the following rule:

$$
v_{t}^{i}= \begin{cases}v_{t-1}^{i}+1 & \text { if } v_{t-1}^{i}+1 \leq \mu\left(v_{t-1}^{i+1}, \delta_{t-1}^{i}\right) \text { and } \xi \leq p_{\text {acc }} \\ v_{t-1}^{i} & \text { if } v_{t-1}^{i}+1 \leq \mu\left(v_{t-1}^{i+1}, \delta_{t-1}^{i}\right) \text { and } \xi>p_{\text {acc }} \\ \mu\left(v_{t-1}^{i+1}, \delta_{t-1}^{i}\right) & \text { otherwise. }\end{cases}
$$

The variable $\xi$ denotes a random number uniformly generated in $[0,1]$. Note that it holds $v_{t-1}^{i}-1 \leq v_{t}^{i} \leq v_{t-1}^{i}+1$. Thereby, acceleration and braking capabilities are limited, and a car changes its speed by at most \pm 1 .

\subsection{Collision free driving}

We will now determine the values of $\mu\left(v_{t}^{i+1}, \delta_{t}^{i}\right)$ for the mNaSch which ensure that there are no collisions between any two cars. (Valid initial configurations for open and periodic boundaries are given in Appendix A.) We need to distinguish between two cases:

1. The vehicle $i$ does not have a predecessor. This is only possible in an open system. In this case the car's speed is only limited by $v_{\max }$, the maximum technical speed of the car. (For the rest of this paper we set $v_{\max }=6$, but our findings remain valid for other choices of $v_{\max }$.)

\footnotetext{
1 This theory is solely based on the meticulous analysis of empirical data.
} 
Table 1

The values of the function $\mu\left(v_{t}^{i+1}, \delta_{t}^{i}\right)$ for $v_{\max }=6$.

\begin{tabular}{|c|c|c|c|c|c|c|c|c|c|c|c|c|c|c|c|c|c|c|c|c|c|c|}
\hline \multirow[t]{2}{*}{$v_{t}^{i+1}$} & \multicolumn{22}{|c|}{$\delta_{t}^{i}$} \\
\hline & 1 & 2 & 3 & 4 & 5 & 6 & 7 & 8 & 9 & 10 & 11 & 12 & 13 & 14 & 15 & 16 & 17 & 18 & 18 & 20 & 21 & $\geq 22$ \\
\hline 0 & 0 & 1 & 1 & 2 & 2 & 2 & 3 & 3 & 3 & 3 & 4 & 4 & 4 & 4 & 4 & 5 & 5 & 5 & 5 & 5 & 5 & 6 \\
\hline 1 & 0 & 1 & 1 & 2 & 2 & 2 & 3 & 3 & 3 & 3 & 4 & 4 & 4 & 4 & 4 & 5 & 5 & 5 & 5 & 5 & 5 & 6 \\
\hline 2 & 1 & 1 & 2 & 2 & 2 & 3 & 3 & 3 & 3 & 4 & 4 & 4 & 4 & 4 & 5 & 5 & 5 & 5 & 5 & 5 & 6 & 6 \\
\hline 3 & 2 & 2 & 2 & 3 & 3 & 3 & 3 & 4 & 4 & 4 & 4 & 4 & 5 & 5 & 5 & 5 & 5 & 5 & 6 & 6 & 6 & 6 \\
\hline 4 & 3 & 3 & 3 & 3 & 4 & 4 & 4 & 4 & 4 & 5 & 5 & 5 & 5 & 5 & 5 & 6 & 6 & 6 & 6 & 6 & 6 & 6 \\
\hline 5 & 4 & 4 & 4 & 4 & 4 & 5 & 5 & 5 & 5 & 5 & 5 & 6 & 6 & 6 & 6 & 6 & 6 & 6 & 6 & 6 & 6 & 6 \\
\hline 6 & 5 & 5 & 5 & 5 & 5 & 5 & 6 & 6 & 6 & 6 & 6 & 6 & 6 & 6 & 6 & 6 & 6 & 6 & 6 & 6 & 6 & 6 \\
\hline
\end{tabular}

2. The vehicle $i$ does have a predecessor. That means that there is another car driving ahead of vehicle $i$. This is always the case in a closed system. The maximum possible speed of car $i$ at time $t$ depends on (i) the speed of its predecessor and (ii) the distance to its predecessor at time $t-1$.

This is captured by the function $\mu\left(v_{t}^{i+1}, \delta_{t}^{i}\right)$ defined as follows:

$$
\mu\left(v_{t}^{i+1}, \delta_{t}^{i}\right)=\min \left\{\left\lfloor\frac{1}{2} \sqrt{8 \delta_{t}^{i}-7+4 v_{t}^{i+1}\left(v_{t}^{i+1}-1\right)}-\frac{1}{2}\right\rfloor ; v_{\max }\right\},
$$

where $\lfloor\cdot\rfloor$ denotes the floor function and $\delta_{t}^{i}$ the distance between car $i$ and its predecessor $i+1$ at time $t$.

The values resulting from Eq. (2) are given in Table 1 for various combinations of a vehicle's headway and speed.

(To some extent this approach is comparable to the work of Emmerich and Rank [20]. They investigated an update mechanism which takes into account both a vehicle's space gap and its speed as well. By ignoring the leading vehicle's speed, this mechanism cannot guarantee realistic deceleration rates.)

Theorem 1. It holds for all times $(\forall t \in \mathbb{N})$ that for any two cars $i, j$ with $i<j: x_{t}^{i}<x_{t}^{j}$. This means that there are no collisions at any time.

Proof. From Table 1 we see that it holds

$$
\mu\left(v_{t-1}^{i+1}, \delta_{t-1}^{i}\right)<\delta_{t-1}^{i}+\max \left\{v_{t-1}^{i+1}-1,0\right\}
$$

and therefore (using $\delta_{t-1}^{i}=x_{t-1}^{i+1}-x_{t-1}^{i}$ )

$$
x_{t}^{i} \leq x_{t-1}^{i}+\mu\left(v_{t-1}^{i+1}, \delta_{t-1}^{i}\right)<x_{t-1}^{i+1}+\max \left\{v_{t-1}^{i+1}-1,0\right\} \leq x_{t}^{i+1},
$$

where we assume without loss of generality that $x_{t}^{i}<x_{t}^{i+1} \forall t \in \mathbb{N}$. Therefore, there are no collisions of any two cars for all times $t$.

To understand the vehicle dynamics in our model, let us look at the values of $\mu\left(v_{t}^{i+1}, \delta_{t}^{i}\right)$ in Table 1 . The values of $\mu\left(v_{t}^{i+1}, \delta_{t}^{i}\right)$ represent a "safe" speed when following a vehicle at a distance $\delta_{t}^{i}$ which has the speed $v_{t}^{i+1}$. The first row, for example, provides the speeds which allow a vehicle to come to rest behind a stopped vehicle without exceeding the maximum deceleration rate of one cell per time step. (A vehicle $i$ driving with $v_{\max }$ and $\delta_{t}^{i}=22$ will reduce its speed to $v_{t+1}^{i}=6, v_{t+2}^{i}=5, \ldots, v_{t+7}^{i}=0$. The total distance traveled in this case equals 21 leading to $\delta_{t+7}^{i}=1$.) For any possible configuration, the values provided by $\mu\left(v_{t}^{i+1}, \delta_{t}^{i}\right)$ guarantee that a vehicle can come to rest by reducing its speed by -1 in each time step. Therefore, the condition $v_{t-1}^{i}+1 \leq \mu\left(v_{t-1}^{i+1}, \delta_{t-1}^{i}\right)$ in Eq. (1) guarantees that a vehicle accelerates only if it is safe to do so. From this follows the limited acceleration rate: $v_{t}^{i} \leq v_{t-1}^{i}+1$. Only the last condition in Eq. (1), which sets $\mu\left(v_{t-1}^{i+1}, \delta_{t-1}^{i}\right)$ as the new speed $v_{t}^{i}$, may cause a vehicle to decelerate.

Theorem 2. By the definition of the function $\mu(v, \delta)$, the braking capabilities of the cars are limited. This means that the following inequality holds:

$$
\mu\left(v_{t}^{i+1}, \delta_{t}^{i}\right) \geq \mu\left(v_{t-1}^{i+1}, \delta_{t-1}^{i}\right)-1 \quad \forall t \in \mathbb{N} .
$$

Proof. The theorem follows directly from the definition of $\mu\left(v_{t}^{i+1}, \delta_{t}^{i}\right)$ as shown in Table 1.

Altogether, we can conclude $v_{t-1}^{i}-1 \leq v_{t}^{i} \leq v_{t-1}^{i}+1$.

\section{Results}

In this section we will present results from simulations of the mNaSch. To begin our analysis we will present fundamental diagrams for different values of $p_{\text {acc }}$. For the simulation we used a road length of $L=10^{4}$ sites and averaged over $T=L$ time 

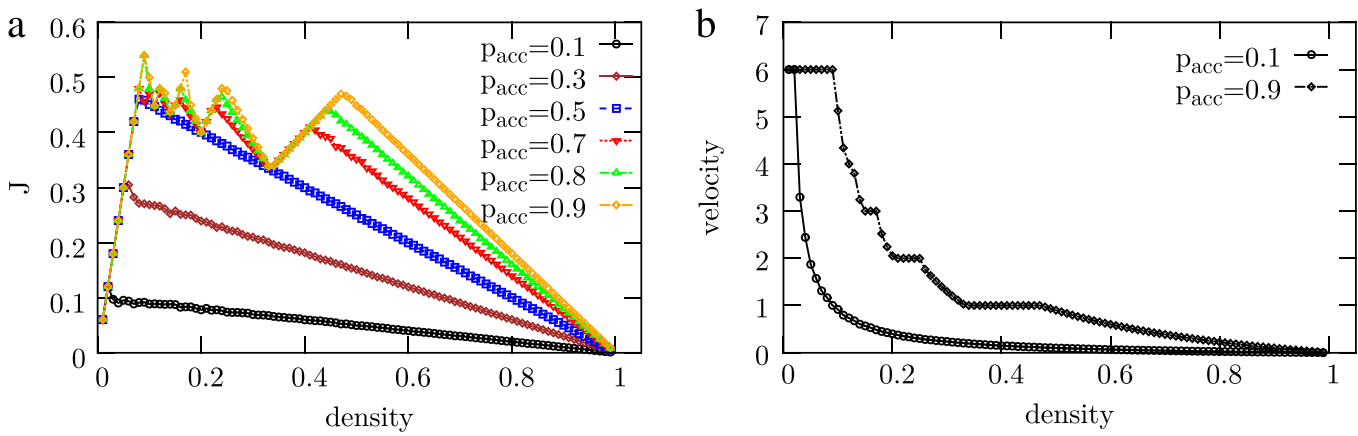

Fig. 1. Fundamental diagram in a closed system (i.e., periodic boundaries) for different values of $p_{\text {acc }}$. As illustrated in Fig. 1(b), the reason for the peaks of the fundamental diagrams for $p_{\text {acc }}>0.5$ are density-regions where the speed remains (nearly) constant.

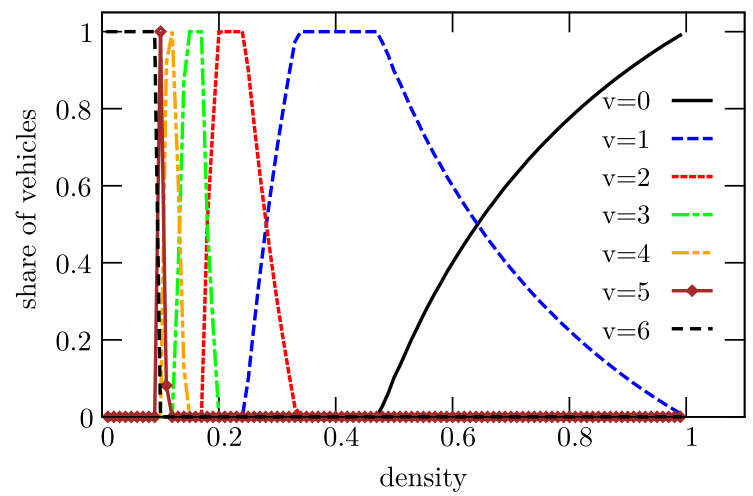

Fig. 2. The distribution of vehicles' speed measured after a relaxation time of $10^{5}$ time steps $\left(p_{\text {acc }}=0.9\right.$ ). For wide density-regions, we find that all vehicles travel with the same speed. Of course, this results in the plateaus observed in Fig. 1(b).

steps after a relaxation time of $10 T$. Densities $0.01 \leq \rho \leq 1$ were simulated in steps of 0.01 for several values of $p_{\text {acc }}$. The fundamental diagrams are shown in Fig. 1.

Similar to the NaSch, the traffic flow increases rapidly until a certain density $\rho_{p_{\text {acc }}}$ is reached and decreases afterwards. The reason is that for densities $\rho<\rho_{p_{\text {acc }}}$ all vehicles can accelerate to the maximal speed $v_{\max }$. Therefore, the flow rate $J$ is given by $J=\rho \cdot v_{\max }$. At higher densities, however, the behavior deviates from that of the NaSch: for $p_{\text {acc }}>0.5$ the flow rate does no longer decrease monotonically with an increasing density, but shows distinct peaks. A comparison with Fig. 1(b) reveals that the formation of the peaks is due to density regions, where the vehicles' average speed is practically independent from the density. The reason of this the major difference between the NaSch and the mNaSch is that the mNaSch converges to stable states where all vehicles move with the same speed $v$ (or with two different speeds $v$ and $v-1$, as we will see later). We will refer to the first case as "speed-synchronized flow". When varying the system's density, the latter case can be regarded as a transition state between two speed-synchronized flows with speeds $v$ and $v-1$.

In Fig. 2 one can see distribution of vehicle speeds for a given density. It is easy to understand that an increase of the vehicle density lowers the share of fast driving vehicles; as the average gap between vehicles decreases with more vehicle on the road, the vehicles have to slow down to conserve collision-freeness.

\subsection{Spatiotemporal dynamics}

The synchronization between vehicles becomes very clear by analyzing the spatiotemporal dynamics. Therefore, Fig. 3 shows the vehicles' dynamics for $p_{\text {acc }}=0.7$. The vehicles started from a compact jam with speed zero. The synchronization of speeds manifests itself by the cancellation of all but one or (at most) two distinct speeds. In the case where two speeds predominate simultaneously, wave-like patterns form which separate groups of vehicles driving with different speeds (Fig. 3(d)).

We may speak of these groups as clusters or platoons of vehicles. In contrast to an earlier model [21], vehicles within such a platoon keep a realistic (i.e., non-zero) safety headway to their predecessor. This can be seen in Fig. 3(c) and (d) which show the distributions of headways obtained from Fig. 3(a) and (b), respectively.

It is important to note that the two patterns found in Fig. 3(a) and (b) are characteristic for all densities and various initial conditions (e.g., homogeneous or random placement of cars). Another important aspect is that due to the model's stochastic acceleration (instead of stochastic deceleration) jams (so-called phantom jams) do not occur spontaneously in the mNaSch. 

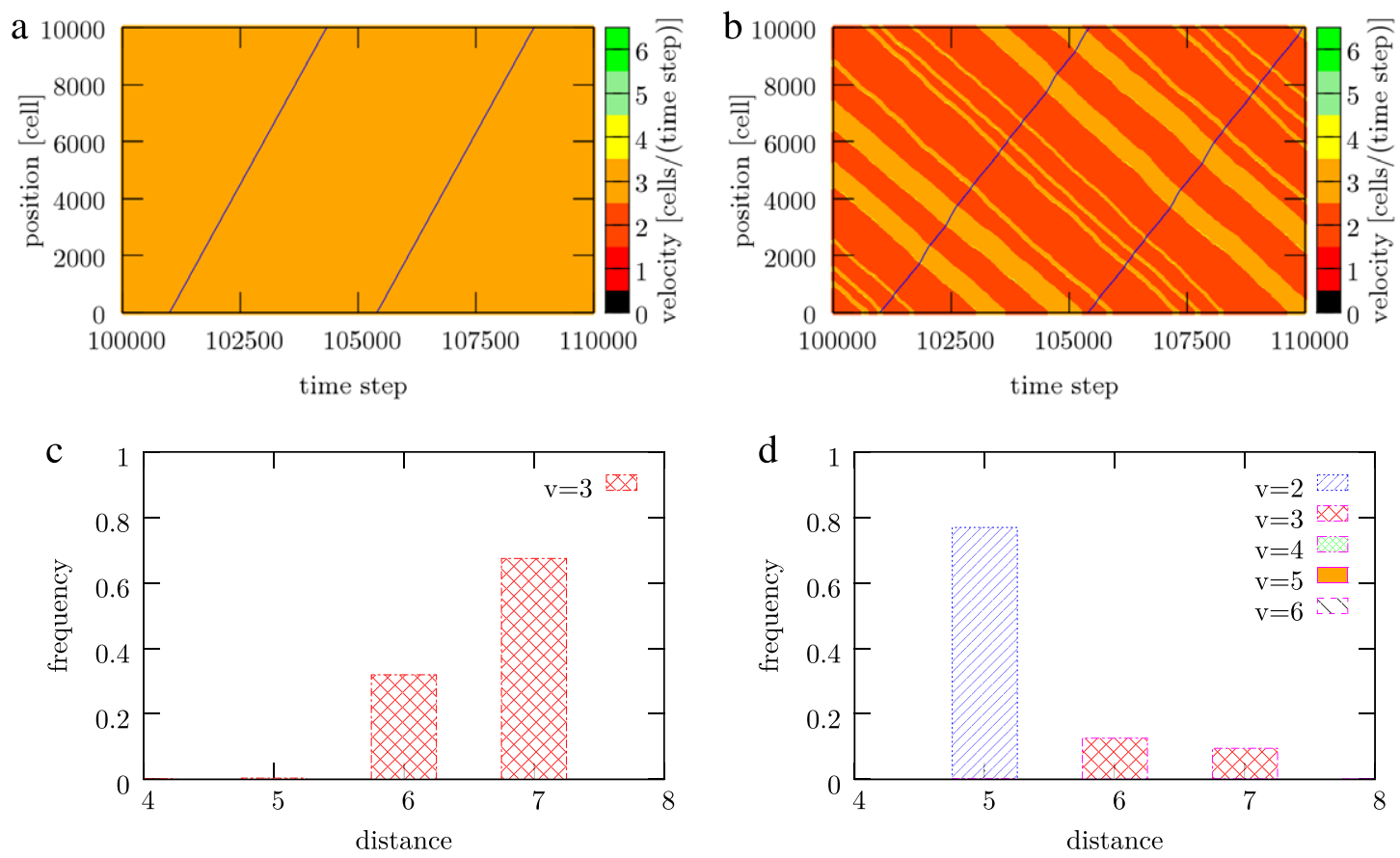

Fig. 3. Spatiotemporal configurations for vehicle densities of (a) $\rho=0.2$ and (b) $\rho=0.25$ ( $p_{\text {acc }}=0.7$ ). Blue lines show the trajectories of two vehicles passing position 0 at times $t=101000$ and $t=105400$, respectively. The corresponding headway distribution is given in figures (c) and (d), respectively These distributions also discriminate between the distance of the vehicles and their speeds. At $\rho=0.2$ all vehicles travel with $v=3$, and the distance $\delta$ to their predecessor measures either $\delta=6$ or $\delta=7$. At $\rho=0.25$, practically all vehicles travel with a speed of $v=2$ or $v=3$. As one can see, the slower vehicles also have a lower headway.

\section{Conclusion and perspectives}

The focus of this work was laid on a comparison of the Nagel-Schreckenberg model and our modified version (mNaSch). We showed by means of simulations that the NaSch converges to either a steady state of speed-synchronized flow or a steady state with only two different speeds. We regarded the latter case as a transition state between two states of speedsynchronized flow. We found differences between the fundamental diagrams of the two models. The fundamental diagrams of the mNaSch model have been shown to be more complex than those of the NaSch. Traffic flow is not simply increasing until a certain density is reached and decreases then, but it is moving in waves with the peak-values decreasing in density. The two principal differences between the NaSch and the mNaSch are that (i) in the mNaSch braking capabilities are limited and (ii) vehicles accelerate with a certain probability whereas in the NaSch vehicles decelerate at random.

It is obvious that the latter causes the convergence towards steady states.

To fully understand the dynamics of the mNaSch it would be helpful to examine the convergence to stable states in an analytical way. It might also be interesting to extend the model: there could be different maximal technical speeds for the cars. This would lead to a non-linear relationship of density and traffic flow before the density is reached.

\section{Appendix. Initial conditions guaranteeing collision free driving}

The proof of collision free driving in Section 2.1 requires, of course, that the road's previous configuration was free of collisions as well. Therefore, we present valid initial configurations for both open and periodic boundaries. Note that the presented configurations are very simple as the focus of this article is on the model's dynamics on a circular road. Especially with respect to open boundaries, the modeling of the boundaries may have a strong impact on the bulk dynamics (e.g., leading to boundary-induced phase transitions [22] or affecting the maximally achievable flow rate [23]).

The case of periodic boundaries, which represent a circular road, is trivial: initially, $N$ vehicles are randomly set on the road, and the initial speed of each vehicle is 0 . Consequently, it holds that $x^{i} \neq x^{j}$ for $i \neq j$ and $\min \left(\delta^{i}\right) \geq 1 \forall i$. Because the road is modeled as a circle, it follows $x_{t}^{i}=\left(x_{t-1}^{i}+v_{t}^{i}\right) \bmod L$ for all later times $t$.

An open system (i.e., open boundaries) represents a bottleneck situation where each car passes through the road only once. New cars enter the road via the left boundary, which requires that the leftmost site $(x=1)$ is empty. In this case a new car labeled $k$ can be inserted with speed $v_{t}^{k}=\min \left\{v_{\operatorname{maxin}}, \mu\left(v_{t-1}^{k+1}, x_{t-1}^{k+1}-1\right)\right\}$ at position $x_{t}^{k}=1+v_{t}^{k}$, where $v_{\text {maxin }}$ denotes the maximum speed of inserted cars. (In our simulations we set $v_{\text {maxin }}=2$.) Afterwards, we apply the rules of motion to the remaining cars (i.e., all but the newly inserted one) and obtain the road's configuration at time $t$. 


\section{References}

[1] Kai Nagel, Michael Schreckenberg, A cellular automaton model for freeway traffic, J. Phys. France I 2 (12) (1992) 2221-2229.

[2] Debashish Chowdhury, Ludger Santen, Andreas Schadschneider, Statistical physics of vehicular traffic and some related systems, Phys. Rep. 329 (2000) $199-329$.

[3] Robert Barlovic, Thorsten Huisinga, Andreas Schadschneider, Michael Schreckenberg, Adaptive traffic light control in the ChSch model for city traffic, in: Traffic and Granular Flow, 2003, pp. 331-336.

[4] Sigurdur Hafstein, Roland Chrobok, Andreas Pottmeier, Joachim Wahle, Michael Schreckenberg, Cellular automaton modeling of the autobahn traffic in North Rhine-Westphalia, in: Proc. 4th MATHMOD, Vienna, 2003.

[5] Andreas Pottmeier, Roland Chrobok, Sigurdur Hafstein, Florian Mazur, Michael Schreckenberg, OLSIM: up-to-date traffic information on the web, in: Proc. 3rd IASTED Int. Conf., St. Thomas, 2004

[6] Sven Maerivoet, Bart De Moor, Cellular automata models of road traffic, Phys. Rep. 419 (1) (2005) 1-64

[7] Andreas Schadschneider, Debashish Chowdhury, Katsuhiro Nishinari, Stochastic Transport in Complex Systems: from Molecules to Vehicles, Elsevier Science, Oxford, 2010.

[8] Elmar Brockfeld, Reinhart Kühne, Alexander Skabardonis, Peter Wagner, Toward benchmarking of microscopic traffic flow models, Transp. Res. Rec. 1852 (1) (2003) 124-129.

[9] Florian Knorr, Michael Schreckenberg, On the reproducibility of spatiotemporal traffic dynamics with microscopic traffic models, J. Stat. Mech. 2012 (10) (2012) P10018.

[10] Bhagwant Persaud, Sam Yagar, Russell Brownlee, Exploration of the breakdown phenomenon in freeway traffic, Transp. Res. Rec. 1634 (1998) 64-69.

[11] Robert Barlovic, Ludger Santen, Andreas Schadschneider, Michael Schreckenberg, Metastable states in cellular automata for traffic flow, Eur. Phys. J. B 5 (3) (1998) 793-800.

[12] Wolfgang Knospe, Ludger Santen, Andreas Schadschneider, Michael Schreckenberg, Towards a realistic microscopic description of highway traffic, J. Phys. A 33 (48) (2000) L477-L485.

[13] Florian Knorr, Michael Schreckenberg, The comfortable driving model revisited: traffic phases and phase transitions, J. Stat. Mech. 2013 (7) (2013) P07002.

[14] Boris S. Kerner, The Physics of Traffic, Springer, Berlin, 2004.

[15] Boris S. Kerner, Introduction to Modern Traffic Flow Theory and Control: The Long Road to Three-Phase Traffic Theory, Springer, Berlin, 2009.

[16] Boris S. Kerner, Criticism of generally accepted fundamentals and methodologies of traffic and transportation theory: a brief review, Physica A 392 (21) (2013) 5261-5282.

[17] Boris S. Kerner, Sergey L. Klenov, Dietrich E. Wolf, Cellular automata approach to three-phase traffic theory, J. Phys. A 35 (47) (2002) 9971.

[18] Hyun Keun Lee, Robert Barlovic, Michael Schreckenberg, Doochul Kim, Mechanical restriction versus human overreaction triggering congested traffic states, Phys. Rev. Lett. 23 (2004) 238702.

[19] Boris S. Kerner, Sergey L. Klenov, Michael Schreckenberg, Simple cellular automaton model for traffic breakdown, highway capacity, and synchronized flow, Phys. Rev. E 84 (2011) 046110.

[20] Heike Emmerich, Ernst Rank, An improved cellular automaton model for traffic flow simulation, Physica A 234 (3-4) (1997) 676-686.

[21] Maria Elena Lárraga, Jesús Antonio del Río, Andreas Schadschneider, New kind of phase separation in a ca traffic model with anticipation, J. Phys. A 37 (12) (2004) 3769-3781.

[22] Joachim Krug, Boundary-induced phase transitions in driven diffusive systems, Phys. Rev. Lett. 67 (14) (1991) $1882-1885$.

[23] Robert Barlovic, Torsten Huisinga, Andreas Schadschneider, Michael Schreckenberg, Open boundaries in a cellular automaton model for traffic flow with metastable states, Phys. Rev. E 66 (2002) 046113. 